\title{
PREDICTING THE TRANSIENT STABILITY OF CEPS USING AES METHOD
}

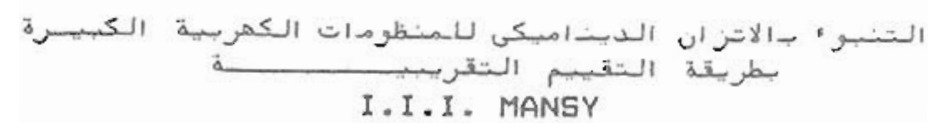

Dept. of Elect.Fower \& Machines

Faculty of Engineer"ing

University of El-Mansoura

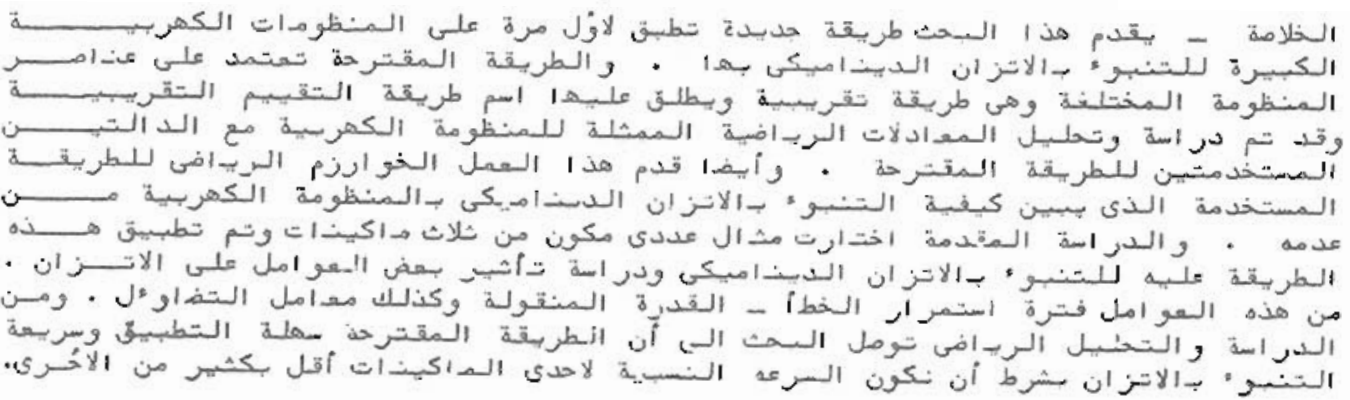

ABSTRACT: Analysis of the stability of power systems following a transient disturbance involves the study of a large set of nonlinear differential equations. A new approach to the quantitative study of the transient stability of complex electric power systems(CEPS) is presented, using approximate evaluation of stability method (AES).

The analytical steps for this method are detailed in this study. Also a constraint, which depends on the relative speed of angle variations, in applying this method for predicting the stability of CEPS is explained.

The present work aimed to study the effect of many factors on the transient stability of a power system. These factors are fault clearing time ( $\left.t_{c}\right)$, damping factor ( $P_{d}$ ) and transmitted power (P).

A suggested flow chart of this method and results of its application to a 3 - machine system are given

\section{INTRODUCTION:}

The problems of power system stability conservation are considered very important. The contral of the transient conditions seems very suitable for stability investigation. The optimal contral problem has been investigated. Hawever, this technique is not able to avercome all the aspects of the problem.This is due to the cascaded growth of disturbance in the power systems.

Therefore, the stability characteristics of the electrical power system (EPS) during transient disturbances are analyzed from stability nature of its mathematical model. A set of differential equations describing EPS is given. These equations are non-linear with constant cofficients under the assumptions of constant emf behind transient reactance and constant input to the machines. 
The stability of linear differential equations can be established from the roots of its characteristic equation [1]. Thus, the stability obtained implies the stability of the system in the entire state space. On the other hand, in a nonlinear system, such an inference is imvalid [2] because its stability depends upon the initial conditions as well as the system parameters. Therefore, a set of initial conditions defining the region of stability has to be found.

This paper deals with the methads, allowing forecasting the mutual stability of EPS at large disturbance. This disturbance occurs in the first stage of transient processes because of the variations in the parameters of EPS operation conditions [3].

\section{NOMENCLATURE}

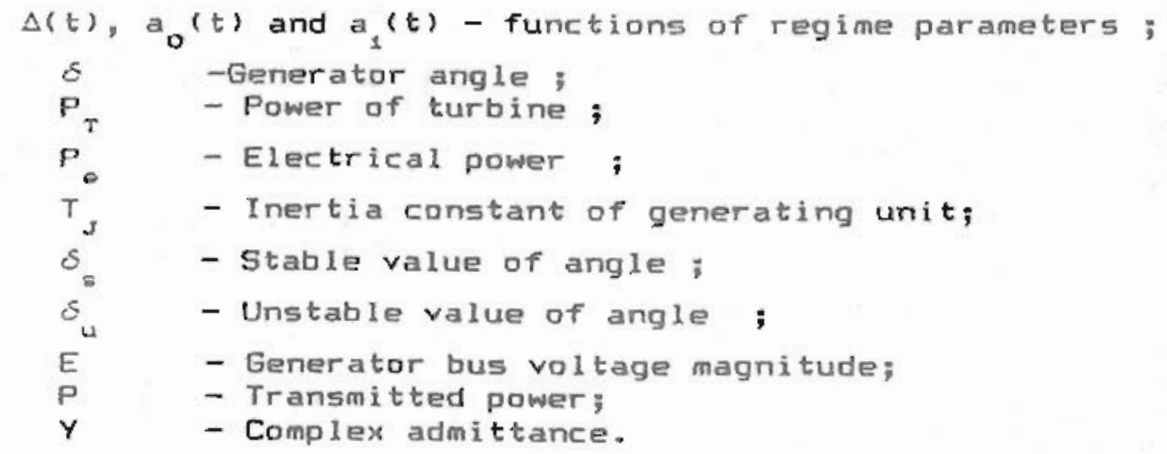

\section{PROBLEM FORMULATION}

The theoritical study of ability to use nonlinear relations $\Delta(t)$ and $a_{i}(t)$, in case of fulfillment of defining conditions of AES method, is demonstrated in ref [4]. Moreover, this referance presents the basic analysis of the mutual stability of electrical inachines in CEPS, but the results of stability study on these systems have not been listed. However, the capability to use these nonlinear relations, which are considered important parameters for this regime, for analyzing the stability of simple power system is

A logical extension of the regime in ref. [4], using AES method for studying mutual stability of CEPS of a radial three-machine system shown in $F$ ig. 1 , is given.It should be noticed, that in radial three - machine system, where one machine returns power to the others, all characteristics of CEPS are satisfied [5]. These characteristics are restricted by the mutual effect of power transmissions, e.g.. the disturbance of stability in one transmissian leading to asynchronous regime for others, existence of stability limit in one power transmission depends on the power which is transmitted by other and similarly. 


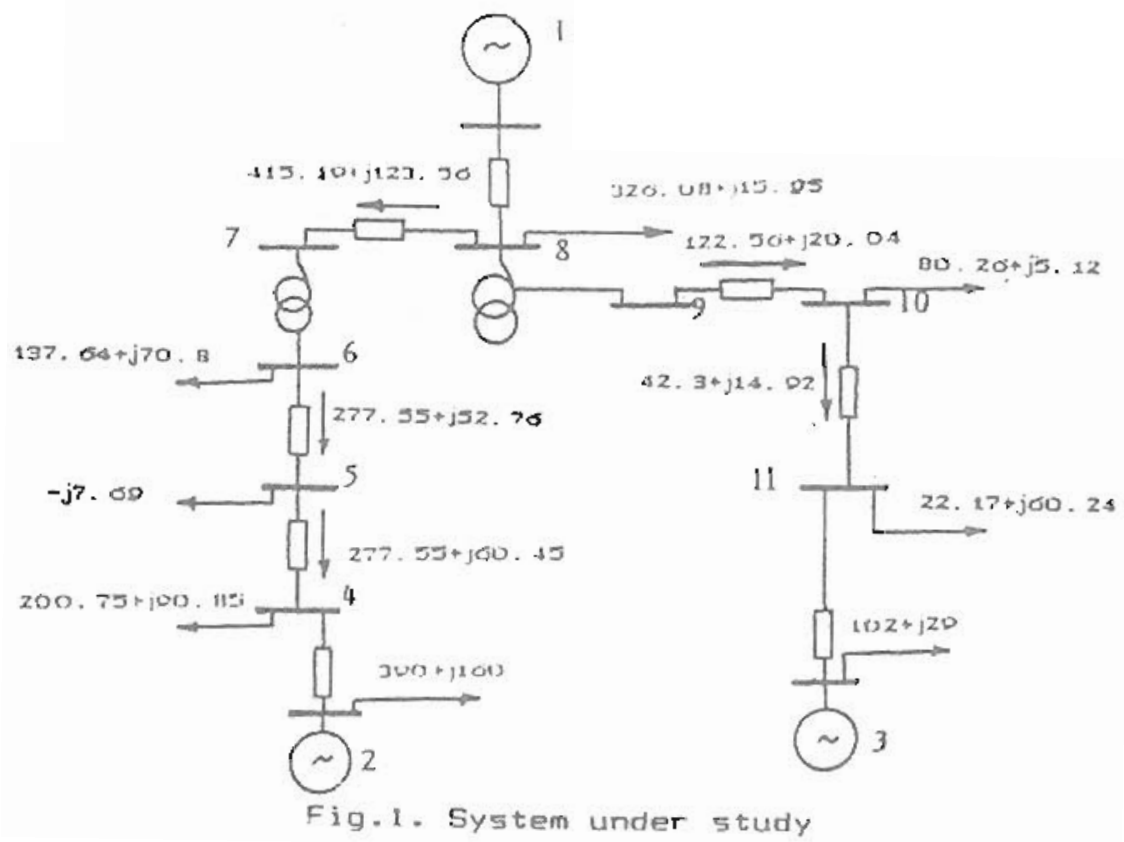

THE PROBLEM SOLUTION

Eelectromechanical transient process in CEPS which consists of three - machine, is described by the following differential equations [6]:

$$
T_{J i} * \ddot{\delta}_{i}+P_{d i} * \bar{\delta}_{i}=P_{T i}-P_{\text {Q }}
$$

Set $P_{d i}=0$

$$
\dot{\delta}_{i}=\frac{P_{I i}}{T_{J i}}-P_{s i} ;
$$

where

$$
\begin{aligned}
& \therefore=1,2,3,- \text { Number of machines ; } \\
& \ddot{\delta}=d^{2} \delta / d t^{2} \text {; } \\
& P_{\text {oi }}=-\frac{1}{T_{j i}}-\left[P_{i t}+\sum_{\substack{j=2 \\
i \neq j}}^{9} P_{i j} \sin \left(\varepsilon_{L j}-a_{i j}\right)\right]
\end{aligned}
$$

Assume the e.m.f, and the torque of turbine are constant, also the loads are represented by constant resistances. Let the relations:

$$
\begin{aligned}
& \delta_{i j}=-\delta_{j i} ; \delta_{i j}=\delta_{i}-\varepsilon_{j} \text { and } \alpha_{i j}=\alpha_{j i} \text { (where } i=1,233 \text {; } \\
& j=1,2 \& 3 \text { and } i \neq j \text { ) in equations (1) \& (2) Then : } \\
& \mathcal{E}_{12}=P_{\text {T12 }}-P_{012} \quad \text { i } \\
& \dot{\delta}_{13}=P_{\mathrm{T} 13}-P_{013}
\end{aligned}
$$




$$
\begin{aligned}
& \text { where }
\end{aligned}
$$

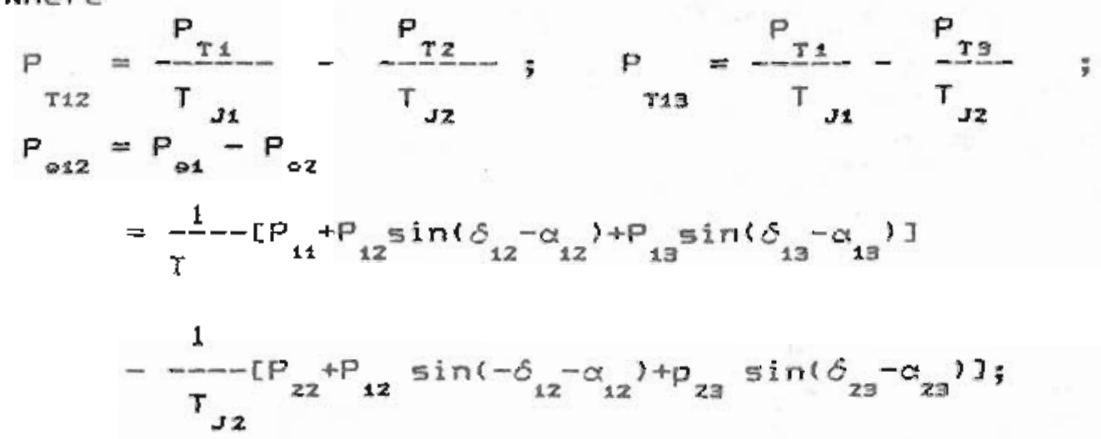

$$
\begin{aligned}
& P_{\text {olg }}=P_{\text {O1 }}-P_{09} \\
& =\frac{1}{T_{J_{1}}}\left(P_{11}+P_{12} \sin \left(\delta_{12}-a_{12}\right)+P_{13} \sin \left(\varepsilon_{13}-a_{13}\right)\right. \\
& -\frac{1}{T_{J 9}}\left[P_{39}+P_{13} \sin \left(-\delta_{13}-a_{13}\right)+P_{23} \sin \left(-\delta_{23}-a_{23}\right)\right] .(6)
\end{aligned}
$$

Similarly, equations using $\delta_{23} ; \delta_{23}$ or $\delta_{13}, \delta_{32}$ can be formulated. In the following, the suggested method is applied for study of mutual stability between first and second machines. In addition the analysis can be extended to study the mutual action between any two machines of system under study.

The nonlinear relations due to performance of variation can be formulated as follows:

$$
\begin{aligned}
& \Delta(t)=\left(\dot{S}_{12}\right)^{2}+\left(\varepsilon_{12}\right)^{2}-\frac{d P}{d \delta} \frac{01 z}{12} ; \\
& \left.a_{1}(t)=-\dot{\delta}_{12}\right)^{2} \frac{d^{2} p_{12} l z}{d \delta_{12}^{2}}, \Delta(t)
\end{aligned}
$$

where

$$
\dot{\delta}=--\frac{d s}{d t}-
$$

The derivative of function $P_{612}$ with respect to angle $\delta_{22}$ can be obtained using parametrical differentiation method [7]. Noticing that:

$$
\delta_{23}=\delta_{13}-\delta_{12} ; \quad \dot{\delta}_{23}=\dot{\delta}_{13}-\dot{\delta}_{12} ; \ddot{\delta}_{23}=\ddot{\delta}_{13}-\ddot{\delta}_{12}
$$

and also

$\dot{\delta}_{23} \dot{\delta}_{12}-\dot{\delta}_{12} \dot{\delta}_{23}=\dot{\delta}_{12} \ddot{\delta}_{13}-\ddot{\delta}_{13} \dot{\delta}_{12}$

Substituting from eqn. (9) in equation (5), 


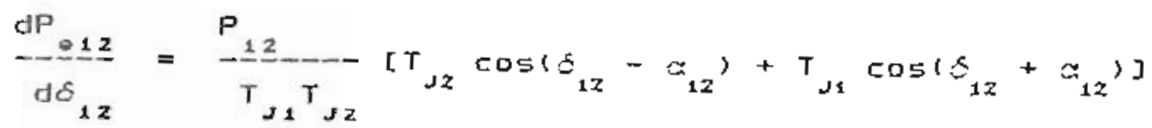

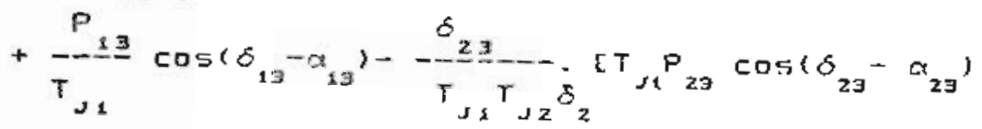

$$
\begin{aligned}
& -T_{J 2} P_{13} \cos \left(\delta_{13}-\alpha_{13} 13 ;\right.
\end{aligned}
$$

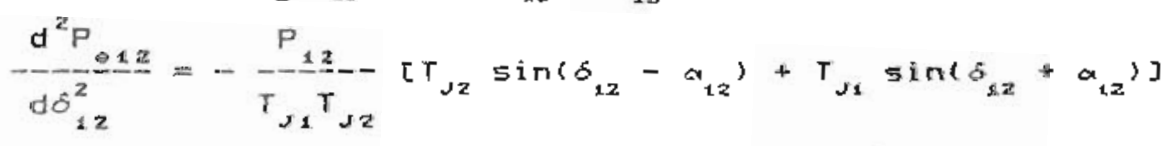

$$
\begin{aligned}
& -P_{13}\left(\delta_{13}\right)^{2} \sin \left(\delta_{13}-\alpha_{13}\right)+\frac{P_{23}}{T_{11}} \delta_{12}\left(-\frac{23}{\delta_{2}}\right)^{2} \sin \left(\delta_{29}-\alpha_{29}\right)
\end{aligned}
$$

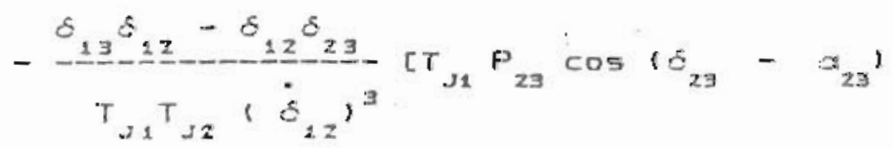

$$
\begin{aligned}
& \left.-T_{s 2} P_{13} \cos \left[\delta_{13}-\alpha_{13}\right)\right] .
\end{aligned}
$$

The sufficient constraint for usability AES method for studying mutual action of rotors of system is available no more at extremum for the equation (5)in every interval of the mutual angle variations possibility $\left[\delta_{12 \pi}\right.$; $\left.\delta_{12 u s}\right] \delta^{2}\left[\delta_{12=}, \delta_{1 z u z}{ }^{3}\right.$

Set equation(10) equal to zero and $a=0$ to simplify the expression. Then, find value of $\delta_{12}$, at which equation (5) has one extremum :

$$
\begin{aligned}
& \cos \delta_{12}=\frac{\dot{\delta}_{23} r_{22} \dot{\delta}_{12}}{T_{23}}\left[T_{21} P_{23} \cos \left(\delta_{23}-\alpha_{23}\right)-r_{22} P_{13} \cos \left(\delta_{23}-a_{13}\right)\right]
\end{aligned}
$$

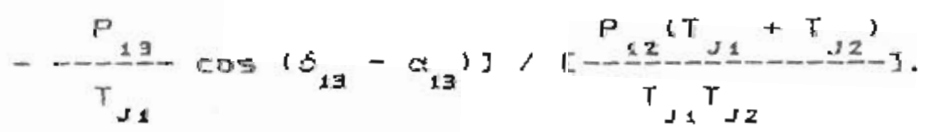

Assume $\dot{\delta}_{29} \ll \delta_{12}$ during transient process- That is in real EPS, for example, when operating conditions of machines 2 and 3 are in phase coincidence "i.e. $5_{23^{2}} 0$ ". Under this condition the first term of equation $\{2$ t is infinitesimal and can be neglected. Then 


$$
\cos \delta_{12}=A \cos \left(\delta_{13}-a_{13}^{\prime} ;\right.
$$

where:

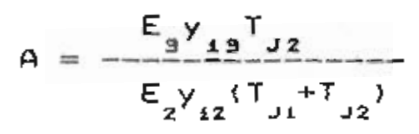

$E_{1} E_{9} Y_{19}=P_{19}$

$$
E_{1} E_{2} Y_{12}=P_{12}
$$

Calculated constraint of yalue $A$ can be considered constant. If relations (12) $\&$ (13) has only one solution through interval $[\Pi k, \Pi(k+1)]$, where $k=i n t e g e r$ number, the value of mutual angle $\delta_{12}$ of function $P_{01 z}$ has anly one extremum in the above interval. Also it can be indicated, that $\varepsilon_{i z}$ has only one extremum in case of the assumptions mode for deduction of constraint (13) and first derivative (10) through the considered intervals.

According to the preceding it can be decided, that the requited constraints in the relations of function $P_{912}$ are satisfied, hence, AES method is used to determine the mutual transient stability in the system under study.

Suggested algorithm of AES method for stability determination between stations during transient process is shown in Fig. (2) in the form of flow - chart.

\section{RESULTS AND DISCUSSION}

The transient operating conditions have been investigated for several factors affecting the stability of power system. These factors are, time elapsed befor clearing the fault, damping cofficient, and the power transmitted by this line. The effect of each factor is numerically computed while the other factors are kept constant. Therefore, the effect of changing the clearing time from 0.37 to 0.38 sec on the stability of the power systems has been revealed.

Assume a double line to ground fault occured in the line connecting between buses 5\& 6. Working voltage of this part is $220 \mathrm{kv}$ while transmitted power is $278 \mathrm{MW}$.

The curves at $t=0.37$ sec., shown in Fig.3, describe the characteristics of mutual angle $\delta_{12}$ and nonlinear relations $\Delta(t)$ \& $a_{1}(t)$ corresponding to the variation of $\delta_{12}=$ Fig.4 shows curves af $s_{12}, \Delta(t)$ \& a $(t) a t t_{c}=0.38$ sec. From Fig.3 function $\Delta(t)$ has positive value during transient process and at $t=0.415 \mathrm{sec}$. $a_{\&}(t)$ has an extremum. Then, mutual stability is conserved. But from Fig. 4 , function d (t) has zero and negative values and $a_{1}(t)$ has an infinite value at $t=0.5$ sec. Then, mutual stability is disturbed. 


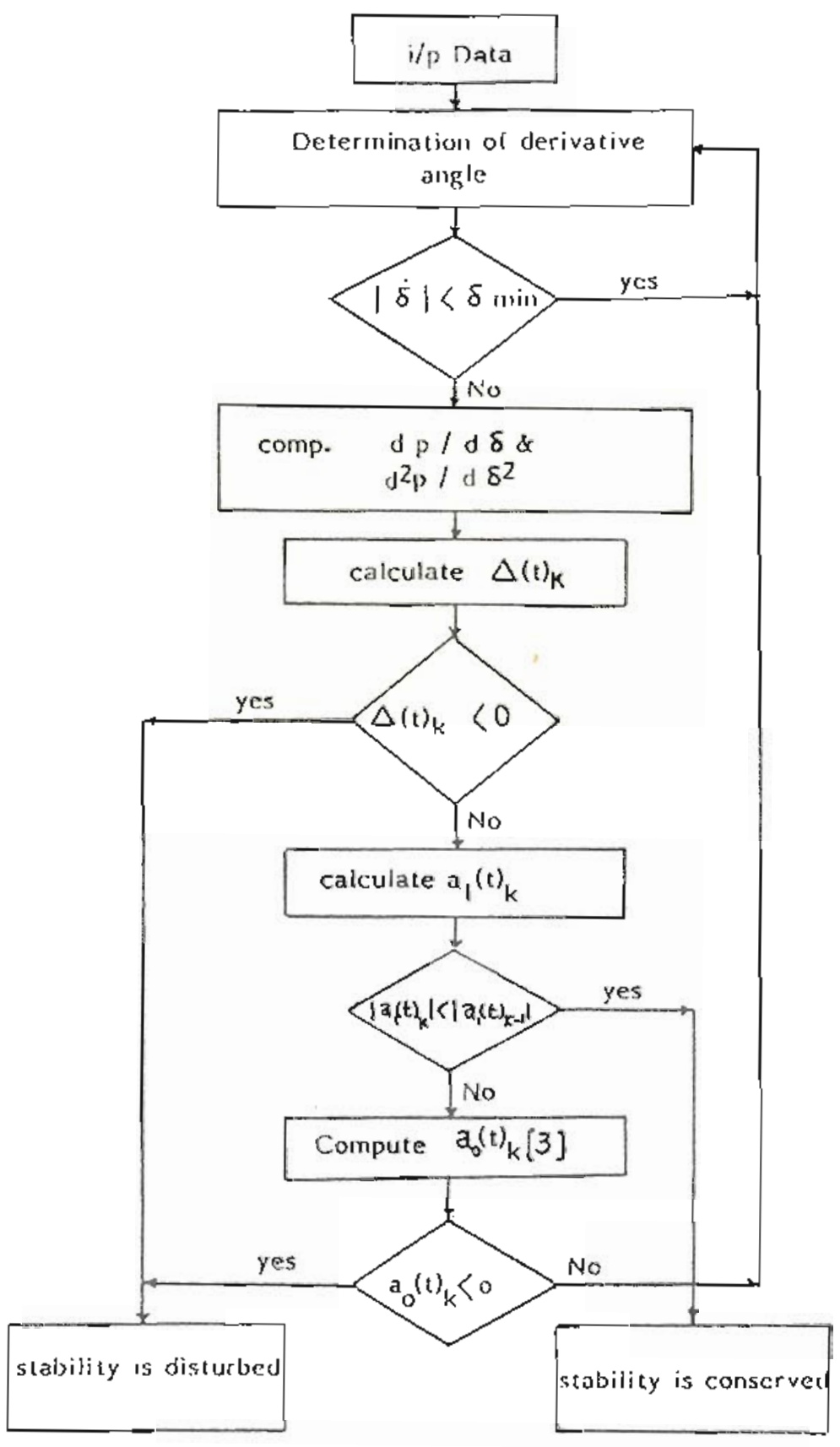

Pig.2. Block diagram ol technique Ior prediction stability of EPS. 


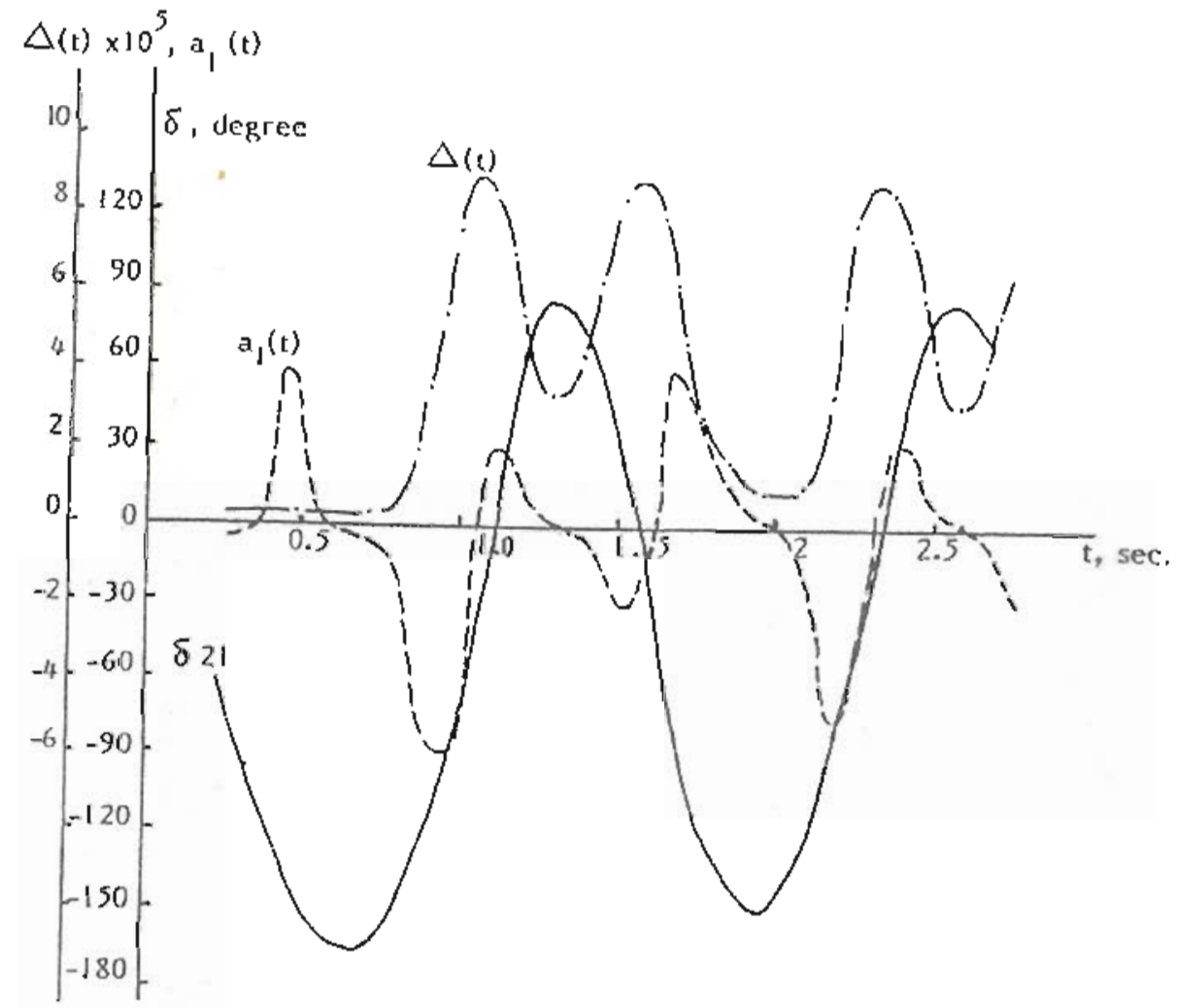

Fig.3. Change of mutual angle and functions $\Delta(t)$ and $a_{1}(t)$ during transient process in the case of $t_{c}=0.37 \mathrm{sec}$. 


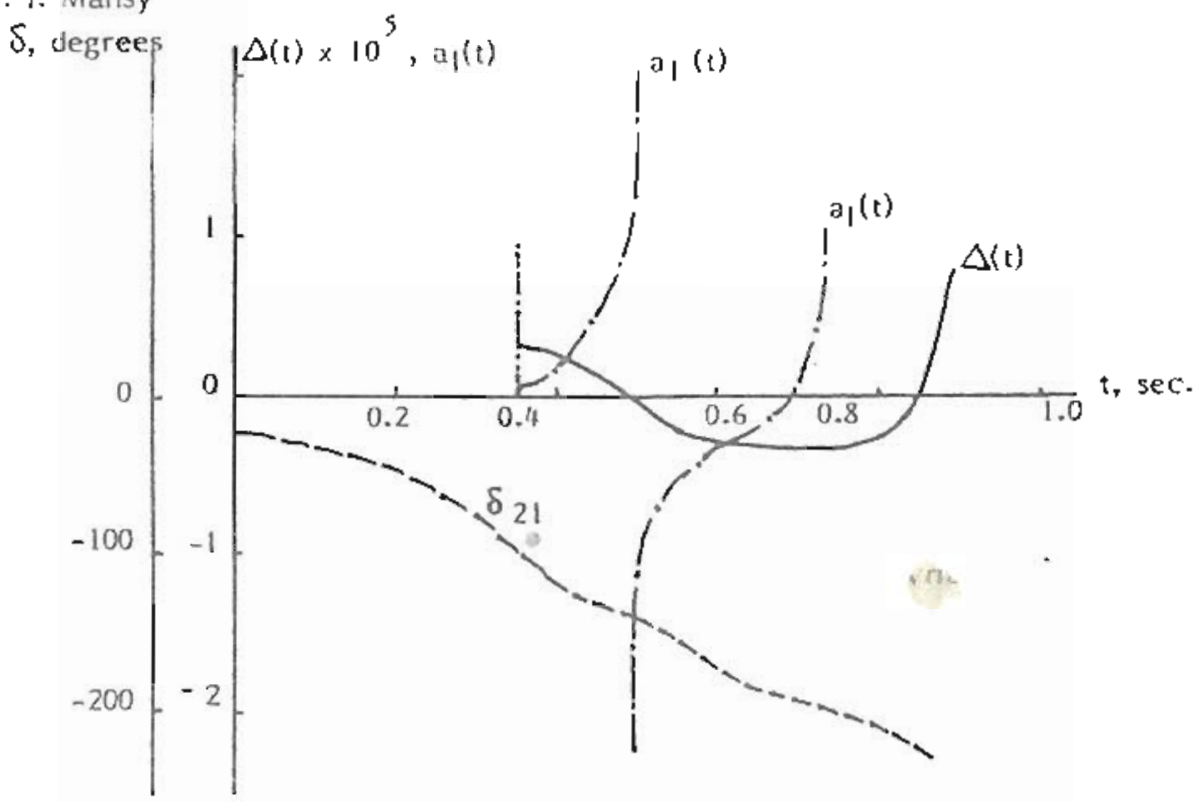

Fig. 4. Change of mutual angle and functions $\Delta(t) a_{1}(t)$ during transient process in the case of $t_{c}=0.38 \mathrm{sec}$.

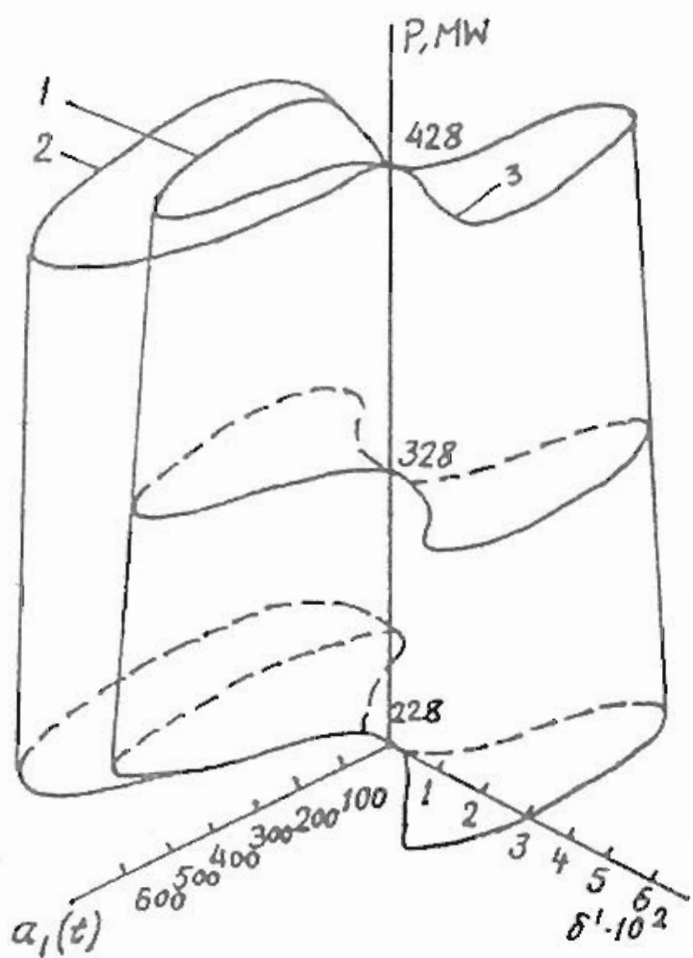

Fig.5. Regions of stability in koordinates $a_{1}(t) \&$ in the case of transmitted power varaitions: $1-p_{d}=0.5 ; 2-p_{d}=5$. and 3 - for the 2 nd half-phase swinging. 
The effect of changing the transmitted power from ees Mw to 428 MW through the 1 ine connecting between buses 635 and also changing the damping factors from 0.5 to 5.0 on the mutual stability of the system under study are shown in Fig.s. Fram this figure at $P_{d}=0.5$. note that loops in second and third quadrants represent the stability regions. But the loops in first and fourth quadrants are considered infinitisimal and indicate trajectory in the second half-phase swinging. Also these loops do not represent in any form the stability 1 imitations for ginen halfphase swinging.

From Fig.5 it can be concluded, that when transmitted power increases bounded region has tendency to get reduced.

\section{EGNCLUEIONS}

In this paper, a new approach for the applicability of AES method is introduced. The approach can be used to predict the mutual transient stability of CEPS. This occurs in the first halfphase swinging under certain constraint. The constraint is that, the relative speed of mutual angle variations must be appreciably less than the other speed.

For real EPS, in the structure of stability regions, AES method can be used for determination of mutual stability, during transient process.

In the existance of important variations of system parameters ( $P$, $P$ ), an indication of the applicability of AEs method can be shown by the recent study.

\section{REFERENCES}

1- A.H. EL-Abiad and K. Nagappan "Transient Stability Regions of Muitimachine Power System", IEEE Trans. Power Apparatus and System, Vol. PAS-BS, No E,Feb. 1966 , PP. $169-179$.

2- H.W. Dommel and N.Sato "Fast Transient Stability Solutions"IEEE Trans. Apparatus and Systems, Vol. PAS - 96, July / Aug. 1972 PP. 253 - 260.

3- V.A. Venikov-and S.N. Asambayer "Advanced Determination of Changes in Power System Parameters in the Control of Transient"-Electrich., 1981, No. 2 PP. 55 - 59.

4- V.A. Venikov and S.N. Asambayev "Approximating Evaluation of Process Stability during its Initial Stages"- Energetica and Transport, 1986, No. 3, PP. $3-9$

5- M.G. Portnoy and R.S. Rabinovich "The Control of EPS for Keeping Stability" - Energia, 1978.

G- V.A. Venikov "Electromechanical Transient Process in Electrical Systems",-Higher Schoo1,1985.

7 A.D. Myskis "Lectures in Higher Mathematics" Mir Publishers, 1975. 